\title{
Electrophysiological Findings in Neuromyelitis Optica Spectrum Disorder
}

\author{
Keiko Ohnari* and Hiroaki Adachi* \\ Department of Neurology, School of Medicine, University of Occupational and Environmental Health, Japan
}

*Corresponding author: Keiko Ohnari and Hiroaki Adachi, Department of Neurology, School of Medicine, University of Occupational and Environmental Health, Japan, keiko-o@med.uoeh-u.ac.jp and hiadachi@med.uoeh-u.ac.jp

\begin{abstract}
Neuromyelitis Optica Spectrum Disorder (NMOSD) has been differentiated from Relapsing Remitting Multiple Sclerosis (RRMS) by clinical, laboratory, and pathological findings, including the presence of the anti-aquaporin 4 antibody. However, it is recognized that patients with NMOSD are hard to differentiate from those with RRMS. Measurement of Evoked Potentials (EPs) is often used to diagnose RRMS and to find subclinical lesions in patients with RRMS, but EPs have not been investigated in detail in patients with NMOSD. Here, we have compared EPs in patients with NMOSD to those with RRMS. Characteristic findings in patients with NMOSD were the absence of both visual evoked potentials and of motor evoked potentials in the lower extremities, while in patients with RRMS there were delays in these potentials. Most patients with NMOSD did not present abnormal subclinical EPs, whereas many patients with RRMS did. None of the patients with NMOSD showed abnormalities in auditory brainstem responses. These data lead us to conclude that NMOSD can be differentiated from RRMS by EP data obtained in the early stages of these diseases.
\end{abstract}

Keywords: Neuromyelitis Optica Spectrum Disorder; Relapsing-Remitting Multiple Sclerosis; Visual Evoked Potentials; Auditory Brainstem Responses; Motor Evoked Potentials

Abbreviations: NMOSD: Neuromyelitis Optica Spectrum Disorder; MS: Multiple Sclerosis; RRMS: Relapsing Remitting Multiple Sclerosis; SPMS: Secondarily Progressive Multiple Sclerosis; PPMS: Primary Progressive Multiple Sclerosis; EP: Evoked Potential; AQP4-Ab: Anti-Aquaporin 4-Antibody; EDSS: Expanded Disability Status Scale; VEP: Visual Evoked Potential; ABR: Auditory Brainstem Response; SEP: Somatosensory Evoked Potential; MEP: Motor Evoked Potential; UE: Upper Extremity; LE: Lower Extremity; CSCT: Central Sensory Conduction Time; TMS: Transcranial Magnetic Stimulation; CMCT: Central Motor Conduction Time; MRI: Magnetic Resonance Imaging

\section{Introduction}

Neuromyelitis Optica Spectrum Disorder (NMOSD) is an autoimmune, inflammatory, necrotic disease with lesions in the optic nerve and spinal cord. It has been challenging to distinguish NMOSD from Relapsing Remitting Multiple Sclerosis (RRMS), an autoimmune, inflammatory, demyelinating disease of the central nervous system. At present, NMOSD is recognized as a different disease from RRMS due to its specific association with the Anti Aquaporin 4 antibody (AQP4-Ab) [1,2]. On the other hand, patients positive for AQP4-Ab that presented with not only optic neuritis and transverse myelitis but also with symptoms of lesions in the brain stem and/or cerebrum have been classified as having NMOSD [3]. Moreover, not all NMOSD patients have serum AQP4-
$\mathrm{Ab}$, and some patients with NMOSD are similar to RRMS in their clinical symptoms and MRI findings [4,5]. Distinguishing NMOSD from RRMS is important because disease-modifying therapies are different for the two diseases. Evoked Potentials (EPs) have been used in patients with RRMS as an important examination for diagnosis. In this review, we summarize the utility of EPs in the differential diagnosis of RRMS and NMOSD.

\section{Evoked Potentials}

An EP is an electrical potential with a specific pattern recorded from a specific part of the central nervous system following a particular stimulus. Visual EPs (VEP) can be used to confirm damage to the visual pathway, including the retina, optic nerve, optic chiasm, 
optic radiations, and occipital cortex [6]. VEPs are induced by a visual stimulation consisting of looking at a checkerboard pattern on a computer screen. When using the standard 10-20 EEG pattern of electrodes VEPs are recorded at the $\mathrm{Oz}$ electrode with reference to the Fz electrode.

The P100 component of the VEP response, which is a positive peak with a delay of about $100 \mathrm{~ms}$ from the stimulus presentation, is of major clinical importance. The P100 originates from the occipital cortex, the first visual field. An abnormal latency or no P100 suggests the presence of a lesion somewhere between the optic nerve and the occipital cortex. Visual pathway dysfunctions anterior to the optic chiasm may be the place where VEPs are most useful for diagnoses. For example, patients with acute severe optic neuritis often lose the $\mathrm{P} 100$ response [7].

The Auditory Brainstem Response (ABR) is used to evaluate the integrity of the auditory pathway up to the midbrain. An ABR is elicited in the auditory nerve within the auditory pathway in the brain stem by a sound stimulation. The response is generated in the cochlea, passes through the cochlear nerve, the cochlear nucleus, the superior olivary complex, the lateral lemniscus, to the inferior colliculus in the midbrain; it then passes on to the medial geniculate body, and finally to the cortex [8]. ABRs to clicks are recorded in the $\mathrm{Cz}$ electrode with reference to the ipsilateral and contralateral ears. This stimulus elicits between five and seven positive waveforms designated by roman numbers. The I, III, and V waveforms are recorded as main peaks and originate from the auditory nerve, nucleus olivaris superior, and the inferior colliculus, respectively. The latencies of these main peaks (I, III, V). and their inter-peak latencies (I-III, III-V, I-V) are commonly studied.

Somatosensory Evoked Potentials (SEP) are recorded from the brain or spinal cord while repeatedly stimulating a peripheral nerve [9]. SEPs are used to evaluate somatosensory pathways at the peripheral, spinal, cortical, and subcortical levels, and have proven their benefit in assessing disorders of the central nervous system more than of peripheral nerve lesions [10]. We obtained Upper Extremity (UE) and Lower Extremity (LE) SEPs by electrically stimulating the median nerve at the wrist and the tibial nerve at the ankle, respectively. Electrodes for recording the SEPs of the UEs were placed on 3 points: Erb's point, the seventh cervical vertebra, and the postcentral scalp. The reference electrode for Erb's point was placed collaterally to Erb's point. The Fz was used as the reference for the other two points. Electrodes for recording the SEPs of the LEs were placed on the twelfth thoracic vertebra and the central scalp. The reference electrodes were placed on the superior border of the iliac crest and the Fz. Peak latencies were measured as N9 (at Erb's point), N13 (at C7), and N20 (over sensory cortex) in the UE tests and as N20 (at Th12) and P37 (over sensory cortex) in the LE tests. The Central Sensory Conduction Time (CSCT) was calculated by subtracting the cervical (N13) and lumbar (N20) latencies from the cortical (N20 and P37) latencies, to give the CSCTs for the UEs and LEs, respectively. We used SEPs to evaluate the deep sensation tract from the peripheral sensory nerve to the cerebral sensory cortex, and calculated CSCTs in RRMS patients.

Motor Evoked Potentials (MEP) are recorded from muscles following Transcranial Magnetic Stimulation (TMS) of the motor cortex. TMS is a non-invasive method suited for investigating the functional integrity of the corticospinal tract using a wire coil generating a magnetic pulse and leading to multiple descending waves in the pyramidal tract and subsequently to a contraction of a specific muscle [11]. We recorded UE and LE MEPs from the abductor pollicis brevis and abductor hallucis muscles, respectively, after TMS of the contralateral motor cortex using a plane, figure eight coil. The intensity of the TMS was set to $10 \%$ above the MEP threshold in the resting condition. To evaluate pyramidal tract function, we calculated the Central Motor Conduction Time (CMCT) using the following formula based on the $\mathrm{F}$ wave:

$C M C T=M E P$ latency $-(M-$ wave latency $+F$-wave latency -1$) / 2$

\section{EPs in RRMS Patients}

Many clinical utilities of EPs in patients with RRMS have been reported [12-14]. Subclinical lesions in the visual, auditory, deep sensation, and pyramidal tracts can be revealed by EPs [15]. Abnormal VEP findings are included in the McDonald Criteria for RRMS [16]. The summed score of the SEP and MEP exhibit high sensitivity and specificity for RRMS and are correlated with the RRMS -related disability-ambulation scale including the Expanded Disability Status Scale (EDSS) [17]. The correlation between abnormal EPs and EDSS was reported to be higher than between conventional MRI and EDSS [18]. In this way, several studies have suggested a prognostic value for EPs in RRMS and an association between EPs and the severity of clinical disability.

\section{EPs in NMOSD Patients}

There have been several reports on EPs in patients with NMOSD $[19,20]$. More patients with AQP4-Ab were reported to show a lack of the P100 on VEP than those without the antibody [20]. In addition, AQP4-Ab positivity and a visually unevoked response were significantly related to the development of severe visual impairment in patients with NMOSD [20]. VEPs in patients with NMOSD were characterized by the absence of a response or a decreased P100 amplitude with normal latency [19]. It was also reported that abnormal MEPs and SEPs of the LEs in NMOSD were related to higher EDSS, making them a good indicator of disability status and relapsing activity in NMOSD patients [21].

\section{Comparisons between NMOSD and RRMS}

We studied the EP findings of NMOSD and RRMS patients [22]. All of the patients with NMOSD were AQP4-Ab-positive and fulfilled the diagnostic criteria for NMOSD [3]. Using the McDonald criteria [23], only patients with confirmed RRMS were evaluated, while those with secondarily progressive MS and primary progressive MS were excluded from the study. All patients with RRMS were AQP4Ab-negative. Patients with NMOSD and RRMS in whom more than 
5 years had elapsed since their time of onset were also excluded from the study. Thus, we analyzed 23 NMOSD patients and 28 with RRMS.

The clinical characteristics of these two groups of patients are summarized in (Table 1). The NMOSD patients were older $(\mathrm{P}<0.001)$ and the ratio of females was higher than those with RRMS $(P=0.044)$. The latencies from onset to diagnosis were similar in the two groups. The main initial symptoms of both patient groups were visual and sensory disturbances. The prevalence of visual disturbances and bladder dysfunction were significantly higher in the NMOSD group; and hiccups and nausea and bladder dysfunction were found only in patients with NMOSD. Other symptoms did not differ significantly between the two patient groups. However, the mean EDSS score at the first examination was higher in the NMOSD group than in the RRMS group ( $\mathrm{P}>0.001)$. At the EP examination, patients with NMOSD showed variable clinical symptoms with EDSS scores ranging from 2 to 8, whereas those with RRMS had EDSS scores ranging from 1 to 3 . While only 3 patients with NMOSD demonstrated the presence of the oligoclonal band in their CSF, $65.2 \%$ of patients with RRMS were positive for it $(P=0.002)$. Patients with NMOSD who had severe muscle weakness presented longitudinally extensive signal abnormalities on MRI, extending from the cervical cord to the thoracic spinal cord. An intramedullary lesion was also observed, involving predominantly the central gray matter.

Table 1: Clinical features and laboratory data of NMOSD and RRMS patients.

\begin{tabular}{|c|c|c|c|}
\hline & NMOSD & RRMS & $P$-value \\
\hline Number of patients & 23 & 28 & \\
\hline Age at onset (years) ${ }^{\mathrm{a}}$ & $51.7 \pm 13.8$ & $29.8 \pm 8.9$ & $<0.001^{* *}$ \\
\hline Sex (men/women) & $2 / 21$ & $10 / 18$ & $0.044^{*}$ \\
\hline Disease duration (months) ${ }^{\mathrm{a}}$ & $10.7 \pm 14.2$ & $13.8 \pm 17.0$ & 0.48 \\
\hline \multicolumn{4}{|l|}{ Initial symptoms } \\
\hline Visual disturbance & $10(43.5 \%)$ & $4(14.3 \%)$ & $0.029^{*}$ \\
\hline Diplopia & $1(4.3 \%)$ & $6(21.4 \%)$ & 0.112 \\
\hline Dysarthria and dysphagia & $1(4.3 \%)$ & $1(3.6 \%)$ & 1.0 \\
\hline Hiccups and nausea & $3(13.0 \%)$ & $0(0)$ & 0.085 \\
\hline Hearing difficulty & $0(0)$ & $2(7.1 \%)$ & 1.0 \\
\hline Facial nerve palsy & $1(4.3 \%)$ & $3(10.7 \%)$ & 0.617 \\
\hline Muscle weakness & $7(30.4 \%)$ & $2(7.1 \%)$ & 0.061 \\
\hline Sensory disturbance & $8(34.0 \%)$ & $15(53.6 \%)$ & 0.093 \\
\hline Bladder dysfunction & $4(17.4 \%)$ & $0(0)$ & $0.035^{*}$ \\
\hline EDSS score at diagnosis ${ }^{a}$ & $4.0 \pm 2.2$ & $2.1 \pm 0.9$ & $<0.001^{* *}$ \\
\hline $\operatorname{CSF} W B C(/ \mathrm{mm} 3)^{\mathrm{a}}$ & $24.3 \pm 58.7$ & $4.7 \pm 5.5$ & 0.098 \\
\hline CSF protein $(\mathrm{mg} / \mathrm{dL})^{\mathrm{a}}$ & $50.5 \pm 27.8$ & $38.9 \pm 21.7$ & 0.113 \\
\hline CSF oligoclonal band & $3 / 20(15.0 \%)$ & $15 / 23(65.2 \%)$ & $0.002^{* *}$ \\
\hline
\end{tabular}

${ }^{a}$ Mean \pm SD; ${ }^{*} P<0.05,{ }^{*} P<0.01$ (NMOSD vs. RRMS; age of onset, disease duration, EDSS score at diagnosis, and CSF findings were compared using the Student's t-test. Initial symptoms were compared between groups using the Fisher exact test). NMOSD: Neuromyelitis Optica Spectrum Disorder, RRMS: Relapsing Remitting Multiple Sclerosis, EDSS: Expanded Disability Status Scale, CSF: Cerebrospinal Fluid, WBC: White Blood Cell

We compared theEP measurementsin asymptomatic anatomical regions of patients with NMOSD and those with RRMS (Table 2). In the VEP, patients with NMOSD presented a delayed P100 latency in only $1(3.8 \%)$ eye without optic neuritis, whereas those with RRMS exhibited a delayed P100 latency in 15 of 39 (38.5\%) eyes without optic neuritis. The one NMOSD patient sometimes noticed pain in her right eye and presented with a reduced critical flicker fusion frequency. However, she was not diagnosed as having right optic neuritis, because she did not perceive any visual disturbance or show visual defects in the Goldmann perimeter.

None of the patients with NMOSD had abnormal ABRs, whereas patients with RRMS presented abnormal findings without hearing disturbance in 9 (20.5\%) ears. Most of the NMOSD patients did not

present abnormal subclinical findings in any of the EPs that we examined (Table 2), and none presented subclinical abnormalities in ABR, SEP, or MEP. By contrast, in our RRMS patients with normal deep sensation, $16.1 \%$ of their UEs and $20 \%$ of their LEs exhibited abnormal CSCTs. In the MEP tests, all RRMS patients without any muscle weakness or hyperreflexia had at least one extremity showing an abnormal CMCT. And, more than half of the RRMS patients $(16 / 28,57.1 \%)$ presented subclinical abnormalities on one or more EP measurement, suggesting that patients with abnormal subclinical EPs are more likely to be diagnosed as having RRMS. We also compared the prevalence of abnormal EPs in symptomatic regions of patients with NMOSD and those with RRMS (Table 3), but there were no differences in the frequencies of abnormal EP findings between the two groups. 
Table 2: Prevalence of abnormal EPs in asymptomatic regions in NMOSD and RRMS patients.

\begin{tabular}{|c|c|c|c|}
\hline \multirow{2}{*}{ EP } & \multicolumn{2}{|c|}{ Proportion (\%) } & \multirow{2}{*}{ P-value } \\
\cline { 2 - 4 } & NMOSD & $15 / 39(38.5 \%)$ & $0.001^{* *}$ \\
\hline VEP (number of eyes) & $1 / 26^{\mathrm{a}}(3.8 \%)$ & $9 / 44(20.5 \%)$ & $0.004^{* *}$ \\
\hline ABR (number of ears) & $0 / 34(0 \%)$ & $9 / 56(16.1 \%)$ & $0.009^{* *}$ \\
\hline UE SEP & $0 / 41(0 \%)$ & $8 / 40(20.0 \%)$ & $0.019^{*}$ \\
\hline LE SEP & $0 / 25(0 \%)$ & $1 / 23(4.3 \%)$ & 1.000 \\
\hline UE MEP & $0 / 17(0 \%)$ & $1 / 18(5.6 \%)$ & 1.000 \\
\hline LE MEP & $0 / 14(0 \%)$ & \\
\hline
\end{tabular}

${ }^{a}$ Numbers after the slashes (/) represent total number of eyes, ears, or limbs examined. ${ }^{*} \mathrm{P}<0.05,{ }^{* *} \mathrm{P}<0.01$ (NMOSD vs. RRMS; Fisher exact test). EP: Evoked Potential, NMOSD: Neuromyelitis Optica Spectrum Disorder, RRMS: Relapsing Remitting Multiple Sclerosis, VEP: Visual Evoked Potential, ABR: Auditory Brainstem Response, SEP: Somatosensory Evoked Potential, MEP: Motor Evoked Potential, UE: Upper Extremity LE: Lower Extremity

Table 3: Prevalence of abnormal EP findings in symptomatic regions in NMOSD and RRMS patients.

\begin{tabular}{|c|c|c|c|}
\hline \multirow{2}{*}{ EP } & \multicolumn{2}{|c|}{ Proportion (\%) } & \multirow{2}{*}{ P-value } \\
\cline { 2 - 4 } & NMOSD & RRMS & 1.000 \\
\hline VEP & $18 / 20^{\mathrm{a}}(90.0 \%)$ & $9 / 9(100 \%)$ & - \\
\hline ABR & $0 / 0(0 \%)$ & $2 / 2(100 \%)$ & - \\
\hline UE SEP & $1 / 5(20 \%)$ & $0 / 0(0 \%)$ & 0.733 \\
\hline LE SEP & $8 / 19(42.1 \%)$ & $7 / 14(50 \%)$ & 0.166 \\
\hline UE MEP & $1 / 11(9.1 \%)$ & $5 / 13(38.5 \%)$ & 0.267 \\
\hline
\end{tabular}

${ }^{a}$ Numbers after the slashes (/) represent total number of eyes, ears, or limbs examined.

EP: Evoked Potential, NMOSD: Neuromyelitis Optica Spectrum Disorder, RRMS: Relapsing Remitting Multiple Sclerosis, VEP: Visual Evoked Potential, ABR: Auditory Brainstem Response, SEP: Somatosensory Evoked Potential, MEP: Motor Evoked Potential, UE: Upper Extremity, LE: Lower Extremity

A summary of our EP results [22] in NMOSD and RRMS patients are shown in the first column of (Tables 4-6). In NMOSD patients, the VEP P100 was more likely to be absent in patients with NMOSD (26.1\%), but more likely to be delayed in patients with RRMS $(45.8 \%)(\mathrm{p}<0.05$; Table 4). All NMOSD patients showed normal ABRs, while in those with RRMS, 10 out of 46 ears (21.7\%) showed an increased latency (I - III interval increased in 3 patients, and III - V interval increased in 7 patients). The prevalence of abnormal UE SEPs in patients with NMOSD was significantly lower than that

Table 4: Comparison of our VEP findings in NMOSD and RRMS patients with those reported by others.

\begin{tabular}{|c|c|c|c|}
\hline \multicolumn{5}{|c|}{ Ohnari et al. [22] } & Watanabe et al. [20] & Neto et al. [19] \\
\hline \multicolumn{5}{|c|}{ NMOSD } \\
\hline VEP (P100) Absent & $12 / 46^{\mathrm{a}}(26.1 \%)^{* *}$ & $11 / 17(64.7 \%)^{*}$ & $18 / 38(47.4 \%)$ \\
\hline Delayed & $7 / 46(15.2 \%)$ & $1 / 17(5.9 \%)$ & $2 / 38(5.2 \%)$ \\
\hline Normal & $27 / 46(58.7 \%)$ & $5 / 17(29.4 \%)$ & $18 / 38(47.4 \%)$ \\
\hline VEP (P100) Absent & $2 / 48(4.2 \%)$ & $20 / 84(23.8 \%)$ & $28 / 84(33.3 \%)^{*}$ \\
\hline Delayed & $22 / 48(45.8 \%)^{* *}$ & $36 / 84(42.9 \%)$ & \\
\hline Normal & $24 / 48(50.0 \%)$ &
\end{tabular}

${ }^{a}$ Numbers after the slashes $(/)$ represent total number of eyes examined. ${ }^{*} p<0.05,{ }^{* *} p<0.01$ (NMOSD vs. RRMS; Fisher exact test). The anti-aquaporin 4 antibody-positive patients reported by Watanabe et al. [20] have been classified as NMOSD. NMOSD: Neuromyelitis Optica Spectrum Disorder, RRMS: Relapsing Remitting Multiple Sclerosis, VEP: Visual Evoked Potential 
Table 5: Comparison of our SEP findings in NMOSD and RRMS patients with those reported by others.

\begin{tabular}{|c|c|c|c|}
\hline & Ohnari et al. [22] & Watanabe et al. [20] & Tsao et al. [21] \\
\hline \multicolumn{4}{|c|}{ NMOSD } \\
\hline UE SEP Absent & $1 / 46^{\mathrm{a}}(2.2 \%)$ & $0 / 12(0 \%)$ & \multirow{2}{*}{$11 / 29(37.9 \%)$} \\
\hline Delayed & $0 / 46(0 \%)$ & $2 / 12(16.7 \%)$ & \\
\hline Normal & $45 / 46(97.2 \%)^{*}$ & $10 / 12(83.3 \%)$ & $18 / 29(62.1 \%)$ \\
\hline LE SEP Absent & 6/44 (13.6\%) & $1 / 12(8.3 \%)$ & \multirow{2}{*}{$12 / 29(58.6 \%)$} \\
\hline Delayed & $2 / 44(4.5 \%)$ & $2 / 12(16.7 \%)$ & \\
\hline Normal & $36 / 44(81.8 \%)$ & $9 / 12(75.0 \%)$ & $17 / 29(41.4 \%)$ \\
\hline \multicolumn{4}{|c|}{ RRMS } \\
\hline UE SEP Absent & 8/56 (14.3\%) & $10 / 60(16.7 \%)$ & \\
\hline Delayed & $1 / 56(1.8 \%)$ & $8 / 60(13.3 \%)$ & \\
\hline Normal & $47 / 56(83.9 \%)$ & $42 / 60(70.0 \%)$ & \\
\hline LE SEP Absent & 7/54 (13.0\%) & $11 / 60(18.3 \%)$ & \\
\hline Delayed & $8 / 54(14.8 \%)$ & $16 / 60(26.7 \%)$ & \\
\hline Normal & $39 / 54(72.2 \%)$ & $33 / 60(55.0 \%)$ & \\
\hline
\end{tabular}

${ }^{a}$ Numbers after the slashes $(/)$ represent total number of extremities examined. ${ }^{*} p<0.05$ (NMOSD vs. RRMS; Fisher exact test). The anti-aquaporin 4 antibody-positive patients reported by Watanabe et al. [20] have been classified as NMOSD. SEP: Somatosensory Evoked Potential, NMOSD: Neuromyelitis Optica Spectrum Disorder, RRMS: Relapsing Remitting Multiple Sclerosis, UE: Upper Extremities, LE: Lower Extremities

Table 6: Comparison of our MEP findings in NMOSD and RRMS patients with those reported by others.

\begin{tabular}{|c|c|c|c|}
\hline & Ohnari et al. [22] & Watanabe et al. [20] & Tsao et al. [21] \\
\hline \multicolumn{4}{|c|}{ NMOSD } \\
\hline EDSS & $4.2 \pm 2.2$ & $5.0 \pm 2.5$ & $5.4 \pm 1.9$ \\
\hline UE MEP Absent & $0 / 28^{\mathrm{a}}(0 \%)$ & $5 / 13(38.5 \%)$ & \multirow{2}{*}{$10 / 26(38.5 \%)$} \\
\hline Delayed & $1 / 28(3.6 \%)$ & $1 / 13(7.7 \%)$ & \\
\hline Normal & $27 / 28(96.4 \%)$ & $7 / 13(53.8 \%)$ & $16 / 26(61.5 \%)$ \\
\hline LE MEP Absent & $4 / 28(14.3 \%)^{*}$ & $4 / 13(30.8 \%)$ & \multirow{2}{*}{$18 / 26(69.2 \%)$} \\
\hline Delayed & $4 / 28(14.3 \%)$ & $3 / 13(23.1 \%)$ & \\
\hline Normal & $20 / 28(71.4 \%)$ & $6 / 13(46.2 \%)$ & $8 / 26(30.8 \%)$ \\
\hline \multicolumn{4}{|c|}{ RRMS } \\
\hline EDSS & $2.1 \pm 0.9$ & $4.0 \pm 2.8$ & \\
\hline UE MEP Absent & $0 / 36(0 \%)$ & $28 / 71(39.2 \%)$ & \\
\hline Delayed & $6 / 36(16.7 \%)$ & $16 / 71(22.5 \%)$ & \\
\hline Normal & $30 / 36(83.3 \%)$ & $27 / 71(38.0 \%)$ & \\
\hline LE MEP Absent & $0 / 36(0 \%)$ & $15 / 71(21.1 \%)$ & \\
\hline Delayed & $15 / 36(41.7 \%)^{*}$ & $25 / 71(35.2 \%)$ & \\
\hline Normal & $21 / 36(58.3 \%)$ & $31 / 71(43.7 \%)$ & \\
\hline
\end{tabular}

aThe numbers after the slashes (/) represent total number of extremities examined. ${ }^{*} p<0.05$ (NMOSD vs. RRMS; Fisher exact test). The anti-aquaporin 4 antibody-positive patients reported by Watanabe et al. [20] have been classified as NMOSD. MEP: Motor Evoked Potentials, NMOSD: Neuromyelitis Optica Spectrum Disorder, RRMS: Relapsing Remitting Multiple Sclerosis, UE: Upper Extremities, LE: Lower Extremities

\section{Features in EPs of NMOSD}

Patients with RRMS exhibit abnormal EPs in the absence of neurological deficits [24], their main lesions are in the sensory and motor tracts and are detected with high sensitivity by EP testing. On the other hand, patients with NMOSD rarely exhibit abnormal EPs in asymptomatic sites. These results may reflect the pathophysiological differences between NMOSD and RRMS in addition to a difference in the locations of their lesions. Previous MRI studies showed that lesions predominantly in the peripheral white matter of the spinal cord, as in RRMS, are the opposite of the situation in NMOSD, which involves lesions in the central gray matter of the spinal cord 
[25]. In addition to differences in lesion location, pathological studies in patients with RRMS showed the presence of insidious and progressive types of white matter damage, such as normalappearing white matter lesions and axonal neurodegeneration [26], which may appear in the early clinical stages, even though RRMS shows a relapsing course with acute inflammatory demyelinating lesions. Thus, asymptomatic white matter lesions are characteristic of RRMS and may be detected by EPs with high sensitivity. On the other hand, inflammatory, necrotizing damage both in the gray and white matter without insidious and progressive demyelination is the main pathogenesis in NMOSD, which may explain why NMOSD shows only a monophasic or relapsing clinical course without a chronic progressive deterioration and may also explain the lack of asymptomatic EP abnormalities.

The VEP, SEP, and MEP findings of previously reported patients with NMOSD and with RRMS are also shown for comparison in (Tables 4-6). Similar to our findings [20] found that a significantly higher number of patients with NMOSD had no VEP P100 than did RRMS patients, whereas more patients with RRMS showed a delayed P100. These results agree with another previous report showing that NMOSD patients presented a loss of P100 [19] (Table 4). The patients with NMOSD may have displayed a high prevalence of the loss of VEP because severe visual impairment has been shown to be associated with the presence of both anti-AQP4 Ab and loss of VEP [20]. Furthermore, the NMOSD patients showed absent LE MEPs, while those with RRMS presented delayed CMCT. Thus, the LE MEP results were compatible with the VEP results. The patients with NMOSD who showed a loss of MEPs had severe muscle weakness and longitudinally extending spinal cord lesions on MRI.

Tsao et al. [21] reported that MEPs in the LEs were a good indicator for disability status in NMOSD patients whose lesions were in the thoracic spinal cord [21]. In addition, recent studies using diffusion tensor imaging also showed significant white matter involvement combined with gray matter involvement in the spinal cords of NMOSD patients, which was not visible on conventional MRI, and such involvement was associated with the clinical disabilities of patients with NMOSD $[27,28]$. These studies suggest the presence in NMOSD patients of greater diffuse damage in the spinal cord than can be detected by conventional MRI. Therefore, measurement of EPs may reflect the diffuse damage and pathological severity more accurately than conventional MRI. Both Watanabe et al. [20] and Tsao et al. [21] reported that NMOSD patients more frequently presented abnormal MEPs than we observed in our study [22] (Table 6). However, there was no significant difference in their MEP findings between NMOSD and MS patients [20]. The higher average EDSS of patients with NMOSD may correspond to the abnormal MEPs. Although we found significantly more normal UE SEPs in NMOSD patients than in RRMS patients, Watanabe et al. found no significant differences between NMOSD and MS in UE and LE SEPs (Table 5). But SEPs and MEPs in the LE were more affected than in the UE in both patients with NMOSD and those with MS.

While few patients with NMOSD presented abnormal ABRs, delayed ABRs were observed in $21.7 \%$ of patients with RRMS [22]. Abnormal ABRs were frequently seen in most patients with RRMS who had normal hearing or mild hearing loss [29]. In contrast, abnormal ABRs were not detected in NMOSD patients without auditory symptoms, even in the presence of medullary symptoms such as Intractable Hiccups and Nausea (IHN) in this study. IHN was also found in $17 \%$ of patients with NMOSD but in none of the patients with RRMS [30]. MRI showed that in those patients with NMOSD, the medullary lesions involved the area postrema and the nucleus tractus solitaris. In NMOSD patients with anti-AQP4 antibodies and brainstem lesions, the anatomical pathway involved in ABRs might be preserved due to their unique pathophysiological background, although there is one report on ABRs in patients with NMOSD, in which the ABRs were severely attenuated due to hearing loss without brainstem lesions as determined by MRI [31]. Therefore, it may be a critical point for the differentiation of NMOSD from RRMS that patients with RRMS likely present abnormal ABRs even in the early stage of the disease, whereas patients with NMOSD do not.

\section{Conclusion}

While nearly all of our NMOSD patients exhibited normal subclinical EP findings, more than half of the RRMS patients exhibited abnormal subclinical EP findings. However, significantly more NMOSD patients were lacking P100 VEPs and LE MEPs compared with the RRMS patients, suggesting that patients with NMOSD have severe clinical symptoms compared to those with RRMS. Because these EP findings appear to be characteristic in patients with NMOSD and RRMS, EPs may enable us to differentiate patients with NMOSD from those with RRMS in the early stage of the disease.

\section{References}

1. Lennon VA, Wingerchuk DM, Kryzer TJ, Pittock SJ, Lucchinetti CF, et al. (2004) A serum autoantibody marker of neuromyelitis Optica: distinction from multiple sclerosis. Lancet 364(9451): 2106-2112.

2. Wingerchuk DM, Lennon VA, Pittock SI, Lucchinetti CF, Weinshenker BG (2006) Revised diagnostic criteria for neuromyelitis Optica. Neurology 66(10): 1485-1489

3. Wingerchuk DM, Lennon VA, Lucchinetti CF, Pittock SJ, Weinshenker BG (2007) The spectrum of neuromyelitis Optica. Lancet Neurol 6(9): 805815.

4. Siritho S, Apiwattanakul M, Nakashima I, Takahashi T, Fujiwara K, et al. (2014) Features of anti-aquaporin 4 antibody-seronegative Thai patients with neuromyelitis Optica spectrum disorders: A comparison with seropositive cases. J Neurol Sci 341(1-2): 17-21.

5. Flanagan EP, Weinshenker BG, Krecke KN, Lennon VA, Lucchinetti $\mathrm{CF}$, et al. (2015) Short myelitis lesions in aquaporin-4-IgG-positive neuromyelitis Optica spectrum disorders. JAMA Neurol 72(1): 81-87. 
6. Flora Hammond, Lori Grafton (2011) Visual Evoked Potentials. Encyclopedia of Clinical Neuropsychology. Springer Pp.2628.

7. Aminoff Michael, Braunwald Eugene, Fauci Anthony S, Kasper Dennis L, Hauser Stephen L, et al. (2001) Electrophysiologic Studies of the Central and Peripheral Nervous Systems. Harrison's Principles of Internal Medicine (15 ${ }^{\text {th }}$ ed's). Evoked Potentials, McGraw-Hill, USA, Pp.357.

8. Musiek FE, Baran JA (2007) The Auditory system. MA: Pearson Education, Boston, USA.

9. McElligott Jacinta (2011) Somatosensory Evoked Potentials. Encyclopedia of Clinical Neuropsychology, Springer: 2319-2320.

10. Groppa S, Oliviero A, Eisen A, Quartarone A, Cohen LG, et al. (2012) A practical guide to diagnostic transcranial magnetic stimulation: Report of an IFCN committee. Clin Neurophysiol 123(5): 858-882.

11. Kallmann BA, Facklmann S, Toyka KV, Rieckmann P, Reiners K (2006) Early abnormalities of evoked potentials and future disability in patients with multiple sclerosis. Mult Scler 12(1): 58-66.

12. Schlaeger R, Schindler C, Grize L, Dellas S, Radue EW, et al. (2014) Combined visual and motor evoked potentials predict multiple sclerosis disability after 20 years. Mult Scler 20(10): 1348-1354.

13. Hume AL, Waxman SG (1988) Evoked potential in suspected multiple sclerosis: diagnosis value and prediction of clinical course. J Neurol Sci 83(2-3): 191-210.

14. Leocani L, Rovaris M, Boneschi FM, Medaglini S, Rossi P, et al. (2006) Multimodel evoked potential to assess the evolution of multiple sclerosis: A longitudinal study. J Neurol Neurosurg Psychiatry 77(9): 1030-1035.

15. Polman CH, Reingold SC, Edan G, Filippi M, Hartung HP, et al. (2005) Diagnostic criteria for multiple sclerosis: 2005 revisions to the “McDonald Criteria”. Ann Neurol 58(6): 840-846.

16. Kiylioglu N, Parlaz AU, Akyildiz UO, Tataroglu C (2015) Evoked potentials and disability in multiple sclerosis: A different perspective to a neglected method. Clin Neurol Neurosurg 133: 11-17.

17. Invernizzi P, Bertolasi L, Bianchi MR, Turatti M, Gajofatto A, et al. (2011) Prognostic value of multimodal evoked potentials in multiple sclerosis: The EP score. J Neurol 258(11): 1933-1939

18. Margaritella N, Mendozzi L, Garegnani M, Colicini E, Gilardi E, et al. (2012) Sensory evoked potentials to predict short-term progression of disability in multiple sclerosis. Neurol Sci 33(4): 887-982.

19. Neto SP, Alvarenga RM, Vasconcelos CC, Alvarenga MP, Pinto LC, et al. (2013) Evaluation of pattern-reversal visual evoked potential in patients with neuromyelitis Optica. Mult Scler 19(2): 173-178.
20. Watanabe A, Matsushita T, Doi H, Matsuoka T, Shigeto H, et al. (2009) Multimodality-evoked potential study of anti-aquaporin-4 antibodypositive and -negative multiple sclerosis patients. J Neurol Sci 281: 3440.

21. Tsao WC, Lyu RK, Ro LS, Lao MF, Chen CM, etal. (2014) Clinical correlations of motor and somatosensory evoked potentials in neuromyelitis Optica. Plos One 9(11): e113631.

22. Ohnari K, Okada K, Takahashi T, Mahune K, Adachi H (2016) Evoked potentials are useful for diagnosis of neuromyelitis Optica spectrum disorder. J Neurol Sci 364: 97-101.

23. Polman CH, Reingold SC, Banwell B, Clanet M, Cohen JA, et al. (2011) Diagnostic criteria for multiple sclerosis: 2010 revisions to the McDonald criteria. Ann Neurol 69(2): 292-302.

24. Groseth GS, Ashman EJ (2000) Practice parameter: The usefulness of evoked potentials in identifying clinically silent lesion in patients with suspected multiple sclerosis (an evidence-based review): Report of the Quality Standards Subcommittee of the American Academy of Neurology. Neurology 54(9): 1720-1725.

25. Kim HJ, Paul F, Lana Peixoto MA, Tenembaum S, Asgari N, et al. (2015) MRI characteristics of neuromyelitis Optica spectrum disorder an international update. Neurology 84(11): 1165-1173.

26. Lassmann H, Brück W, Lucchinetti C (2007) The immunopathology of multiple sclerosis: An overview. Brain Pathol 17(2): 210-218.

27. Rivero RL, Oliveira EM, Bichuetti DB, Gabbai AA, Nogueira RG, et al. (2014) Diffusion tensor imaging of the cervical spinal cord of patients with Neuromyelitis Optica. Magn Reson Imaging 32(5): 457-463.

28. Rocca MA, Agosta F, Mezzapesa DM, Martinelli V, Salvi F, et al. (2004) Magnetization transfer and diffusion tensor MRI show gray matter damage in neuromyelitis Optica. Neurology 62(3): 476-478.

29. Robinson K, Rudge P (1997) Abnormalities of the auditory evoked potentials in patients with multiple sclerosis. Brain 100: 19-40.

30. Misu T, Fujihara K, Nakashima I, Sato S Itoyama Y (2005) Intractable hiccup and nausea with periaqueductal lesions in neuromyelitis Optica. Neurology 65(9): 1479-1482

31. Takanashi Y, Misu T, Oda K, Miyazaki H, Yahata I, et al. (2014) Audiological evidence of therapeutic effect of steroid treatment in neuromyelitis Optica with hearing loss. J Clin Neurosci 21(12): 2249-2251.
OJNBD

DOI: $10.32474 / 0 J N B D .2021 .05 .000203$

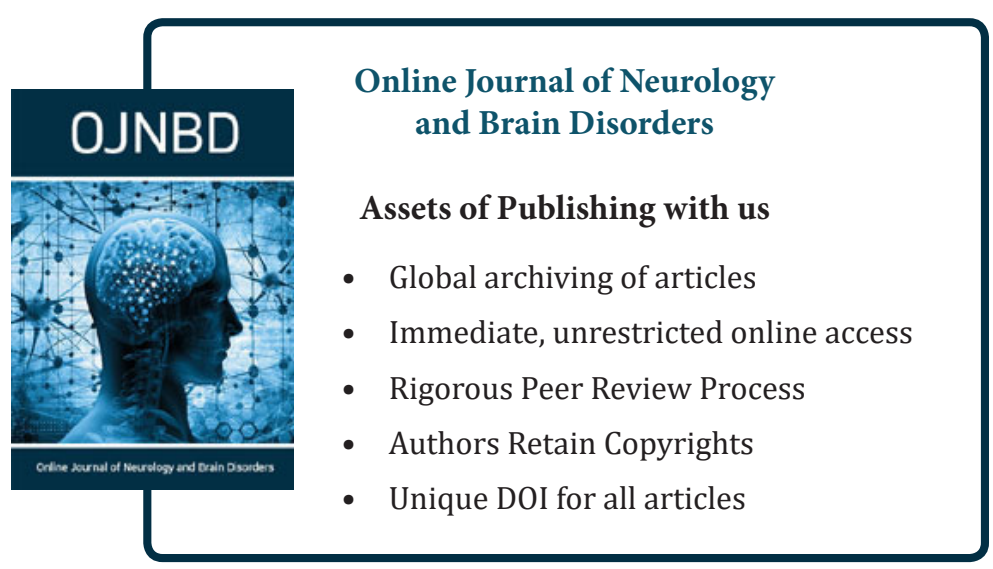

